\title{
Comparison of the Effects of Two Drugs- Etomidate and Propofol on Disorientation and Dementia of the Elderly after Orthopaedic Surgery - A Double-Blind Clinical Trial
} Hossein Ghavipeykar¹, Sayed Hamid Pakzad Moghadam², Alireza Vakilian³ ${ }^{3}$ Ali Sarkoohi ${ }^{4}$, Gholamreza Bazmandegan ${ }^{5}$,
Mohsen Rezaeian ${ }^{6}$

${ }^{1}$ Department of Anaesthesiology, Rafsanjan University of Medical Sciences, Rafsanjan, Iran. ${ }^{2}$ Department of Anaesthesiology, Rafsanjan University of Medical Sciences, Rafsanjan, Iran. ${ }^{3}$ Department of Neurology, Rafsanjan University of Medical Sciences, Rafsanjan, Iran. ${ }^{4}$ Department of Anaesthesiology, Rafsanjan University of Medical

Sciences, Rafsanjan, Iran. ${ }^{5}$ Department of Physiology, Physiology-Pharmacology Research Center, Rafsanjan

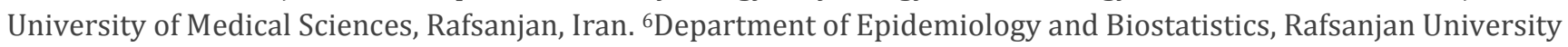
of Medical Sciences, Rafsanjan, Iran.

\section{ABSTRACT}

\section{BACKGROUND}

Elderly are more exposed to cognitive impairment due to the presence of underlying disease and some structural and functional changes in the nervous system. The aim of this study is to compare the effects of two drugs of Etomidate and Propofol on haemodynamics, delirium and dementia of elderly patients after orthopaedic surgery.

\section{METHODS}

In this nonrandomized controlled clinical trial, with the use of sample size formula, 100 patients over 60 years of age who had undergone orthopaedic surgery under general anaesthesia referred to Rafsanjan Ali-Ebn-Abi-Taleb Hospital in 2018 were enrolled in the study by convenience sampling method. After obtaining informed consent, participants were divided into two groups: A) Propofol ( $2 \mathrm{mg} / \mathrm{Kg}$ ) and B) Etomidate $(0.2 \mathrm{mg} / \mathrm{Kg})$ for induction of anaesthesia. Haemodynamic parameters (heart rate, blood pressure, arterial oxygen saturation, amount of bleeding) and cognitive impairment were measured 1 hour before surgery, one day and three days after surgery by MMSE (for checking dementia) and NEECHAM scale (for evaluation of delirium levels). Data was analysed using Independent T-test and chi-square test. The significance level of the tests was considered to be equal to or less than 0.05 .

\section{RESULTS}

Of the 100 patients under study, 56 (56\%) were male and $44(44 \%)$ were female. Hemodynamic change in propofol group was significantly higher than Etomidate group ( $p=0.001$ ) .The amount of bleeding in Propofol group was significantly lower than Etomidate group ( $p=0.047)$. On the first day after surgery, the mean of MMSE score (representing dementia) in the Propofol group was lower than in the Etomidate group $(p=0.043)$ and on the third day after surgery it was more than Etomidate group $(p=0.002)$. On the first day after surgery, the level of delirium in the Propofol group was lower than Etomidate group significantly $(p<0.05)$.

\section{CONCLUSIONS}

The results of this study indicate that the use of Propofol for orthopaedic surgery in elderly patients decreases the chance of delirium and increases the amount of bleeding.

\section{KEY WORDS}

Elderly, Anaesthesia, Propofol, Etomidate, Dementia, Delirium
Corresponding Author: Sayed Hamid Pakzad Maghadam, Medicine College, Khalih Fars Blvd., Rafsanjan, Kerman, Iran. E-mail: pakzadmoghadam@gmail.com

DOI: $10.14260 / \mathrm{jemds} / 2020 / 97$

Financial or Other Competing Interests: None.

How to Cite This Article: Ghavipeykar H, Moghadam SHP, Vakilian $A$, et al. Comparison of the effects of two drugs- etomidate and propofol on disorientation and dementia of the elderly after orthopaedic surgery- a double-blind clinical trial. J. Evolution Med. Dent. Sci. 2020;9(07):428-432, $10.14260 /$ jemds/2020/97

Submission 25-06-2019, Peer Review 22-01-2020, Acceptance 29-01-2020, Published 17-02-2020.

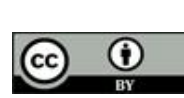




\section{BACKGROUND}

The number of elderly people in the world, including Iran, is increasing. In 2006, more than 5 million people from the country were over 60 years old, representing a total of $7.3 \%$ of the total Iran's subjects.(1) Now $7.8 \%$ of the subjects of Iran is over 65 years and is expected to reach $16 \%$ of the subjects in the coming decades. Researchers considered improvements in health conditions, medical advances, reduction in death rate, and increasing life expectancy, the reasons for increasing longevity and growing subjects of old people.(2) With regard to the growth of the elderly subjects, attention to aging and its issues, including health and welfare issues, is a necessary subject.(3) Elderly problems are associated with an increased risk of illness and complications. Dementia (including neuro-cognitive impairment) one of the most common neurological disorders and one of the most important health threats in the elderly. Dementia is a progressive degeneration of cognitive functions that occurs in a state of complete consciousness. Impairment of memory, attention, thinking, and understanding, and other mental functions, including personality, creativity, judgment, and social behaviour are seen in dementia. Although dementia has several distinct diagnostic criteria, the common element of all is the dramatic decline in social function or occupational dysfunction and a significant drop in the level of individual function.(4) There are various theories regarding the pathophysiology of delirium, including metabolic encephalopathy, hypoglycaemic drug toxicity (especially anticholinergics), surgical stress and increased corticosteroids resulting from it, preoperative hypoxemia, hypotension, anaesthetic type, sleep deprivation and pain, Electrolyte imbalance, old age, Auditory and visual disturbances. $(5,6)$ Delirium, on the other hand, is a common problem and occurs in 15 to $20 \%$ of patients in all hospital wards. This disorder is more common in elderly and patients with a history of cognitive impairment.(7) Delirium is more common in children, elderly, and patients with low socioeconomic status, history of substance abuse, history of neurological disorders, behavioural disorders and dementia. Delirium, which is the most serious postoperative cognitive disorder, is defined as a condition of confusion, which is associated with increased mortality, major disability, prolongation of hospitalization and discharge with long-term care and special rehabilitation facilities.(8) The incidence of bone fractures in the elderly is high due to vision problems, keeping the balance and underlying diseases in the elderly. Subsequently, orthopaedic surgeries are also increased in order to correct fractures in these individuals and use of various anaesthetic drugs. Therefore, it is important to recognize the effects of these drugs on the cognitive functions of the elderly in preventing secondary complications. The aim of this study was to compare the efficacy of two Etomidate and Propofol drugs on dementia and delirium after anaesthesia and orthopaedic surgery in the elderly.

\section{METHODS}

This non-randomized, controlled clinical trial study was approved by ethical committee of Rafsanjani university of medical sciences by code (IR RUMS REC 1394.11) and registered in Iranian Registry of Clinical Trial (IRCT) by code (IRCT2017080835577N1).The subjects of the study included all patients over 60 years of age candidates for orthopaedic surgery who referred to Ali-Ebn Abi Taleb Hospital in Rafsanjan (Kerman, Iran) in 2018. Based on the results of previous studies, $(9,10)$ and the formula $(\alpha=0.05, \beta=0.20$, $\mu 1=25.39, \quad \mu 2=26.8, \sigma 1=\sigma 2=2.41$ ) the sample size was calculated and consequently 92 subjects must be evaluated. Finally, to be sure, 100 people entered in the study. After giving patients the necessary explanations, informed consent was obtained. The inclusion criteria included ASA class I \& II, lack of alcohol addiction, lack of history of mental disorders, MMSE score less than 23, no history of stroke in the 6 months before surgery, and exclusion criteria including patient dissatisfaction during the study. At the beginning, there was a drawing in order to put the first patient in one group (Propofol or Etomidate). Accordingly, the Etomidate was selected. Therefore, the second patient was placed in the Propofol group and this process continued until the last patient. Delirium evaluated by Neecham Confusion Scale (NEECHAM), pain assessed by the visual acuity scale (VAS) and cognitive status assessed by the Mini-Mental State Exam (MMSE). In the first group, Propofol (2 mg/Kg) (Manufacturer B, Brun Melsungen AG Germany) and the second group $(0.2 \mathrm{mg} / \mathrm{Kg})$ (manufacturer Janssen-Cilag 2012) were used. In both groups, other anaesthetic drugs that commonly used in general anaesthesia were calculated and used based on the weight of the patients. The vital parameters including heart rate, respiration rate, SPO2, systolic and diastolic blood pressure before and after anaesthesia and during anaesthesia were evaluated using a digital monitoring system (Saadat model, Iran). After oxygenation, midazolam ( $2 \mathrm{mg})$ and then fentanyl $(2 \mu \mathrm{g} / \mathrm{Kg})$ were injected. Then, Propofol or Etomidate was used for sleeping. Finally, atracurium $(0.5 \mathrm{mg} / \mathrm{Kg})$ was used. After three minutes of ventilation with a mask, the intubation was performed. The Tidal volume $(6-8 \mathrm{cc} / \mathrm{Kg})$ was established and Isoflurane, $\mathrm{N}_{2} \mathrm{O}$, and oxygen were used for continuing anaesthesia. Atracurium repeated every 20 minutes based on the duration of the surgery. The narcotic drug was repeated for the patient's analgesia according to the type of operation and patient's need. During the surgical procedure, the patient's vital signs were measured and recorded at regular intervals. The number of used surgical gauze was recorded. Also, surgical gauzes were weighed before using with a digital scale with accuracy of $0.1 \mathrm{~g}$. The exact volume of fluids, including serums and blood, was also recorded (milliliters). Bloody gauzes were re-weighed immediately after using. The weight difference of gauzes was calculated. Also, the amount of fluids and blood in the suction system was recorded in milliliters, and the difference in volume of intake and outlet of fluids was calculated. The total difference in the weight of gauzes and the difference in volume of fluids were considered as an indicator of bleeding. 


$$
n=\frac{\left(Z_{1-\frac{\alpha}{2}}+Z_{1-\beta}\right)^{2}\left(s^{2}{ }_{1}+s_{2}^{2}\right)}{\left(\mu_{1}-\mu_{2}\right)^{2}}
$$

In this study, we used NEECHAM scale, a nursing instrument for delirium screening, to evaluate the delirium level.(11,12) This scale can be used to evaluate the patient quickly and without interruption in the patient's bedside. The NEECHAM scale has three main items: a) information processing, (score range 0-14) evaluates components of cognitive status: attention and alertness, verbal and motor response, and memory and orientation; b) behaviour (score range of 0-10), evaluates observed behaviour and performance ability: general appearance and posture, sensory-motor performance, and verbal responses; and c) performance (score range 0-16), assesses vital function: vital signs, oxygen saturation level and urinary incontinence. The sensitivity and specificity of the NEECHAM scale were $87 \%$ and 95\% respectively.(11) The Cronbach's alpha coefficient of this scale was calculated to be 0.96 in Jannati et al.(12) For evaluation of cognitive status, a 17-item MMSE test was used, a) orientation (to time and to place) b) registration, c) attention and calculation, d) recall, e) language, f) repetition, g) complex commands. The total score of the MMSE test is 30 that scores of less than 25 indicates cognitive impairment. ${ }^{(13)}$ In addition, Visual analogue scale (VAS) was used to measure of pain. On this scale, there are 10 shapes from the left, "no pain" to the right, "the worst and most severe pain ", and the patient should identifies one of the forms most consistent with his current pain.(14) The cognitive impairments were evaluated using MMSE and NEECHAM scale one hour before surgery, one day and three days after surgery and pain score one day and three days after surgery was evaluated. It should be noted that evaluation of delirium, dementia, pain and evaluation of haematological parameters in different stages of the study was done by anaesthesiology resident that was not aware of the grouping of patients.

\section{Statistical Analysis}

Also, the data analyzer was unaware of the grouping of patients. Finally, the data were analyzed by using SPSS-20 software and Independent T-test, Chi-square. The significance level of the tests was considered to be less than 0.05 .

\section{RESULTS}

Of the 100 surgeries, 50 of the patients (50\%) used Etomidate and $50(50 \%)$ of the patients were treated with Propofol. Of these, 56 males (56\%) and 44 (44\%) were female. The effect of Etomidate and Propofol on the delirium was compared using the NEECHAM scores. On the first day after surgery, there was a significant difference between the two groups in terms of the probability of delirium event, so that delirium was lower in Propofol group $(p=0.01)$. On the third day after surgery, the mean score of NEECHAM was not significantly different between the two groups $(p=0.26)$. The mean MMSE score on the first day after surgery was significant between Propofol and Etomidate $(p=0.043)$, indicating that the first day after surgery, the dementia of the Propofol group was lower than that of the other group. Also, on the third day after surgery, the mean MMSE scores of Propofol and Etomidate groups were significant $(p=0.002)$, with Propofol users having a higher mean MMSE, indicating that the dementia was also lower in the Propofol group in $3^{\text {rd }}$ days. The results showed that the mean amount of bleeding in the Propofol group was lower than Etomidate group $(p=0.047)$ (Table 1). $48 \%$ of patients that treated with Propofol had haemodynamic changes less than $25 \%$ of baseline and $52 \%$ had haemodynamic changes greater than $25 \%$ of baseline, while in the Etomidate group, $96 \%$ had haemodynamic changes less than $25 \%$ of baseline and only $4 \%$ had haemodynamic changes greater than $25 \%$ of baseline. The difference between the two groups was significant in terms of haemodynamic changes $(p<0.001)$. haemodynamic changes followed the use of Propofol more than the Etomidate drug (Table 2).

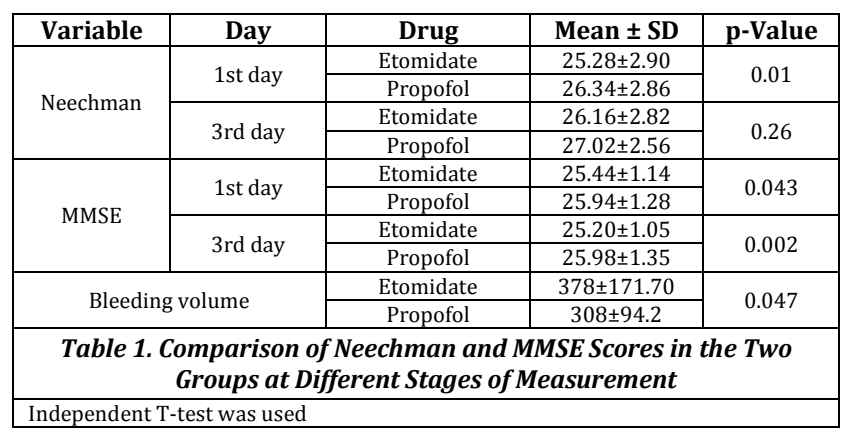

\begin{tabular}{|c|c|c|c|}
\hline \multirow{2}{*}{ Hemodynamic changes } & Propofol & Etomidate & \multirow{2}{*}{ p-value } \\
\cline { 2 - 3 } & No (\%) & No (\%) & \\
\hline$\leq 25 \%$ & $24(48)$ & $48(96)$ & \multirow{2}{*}{$<0.001$} \\
\hline$>25 \%$ & $26(52)$ & $2(4)$ & \\
\hline \multicolumn{3}{|l}{ Table 2. Comparison of haemodynamic Changes } \\
\hline Chi-square was used
\end{tabular}

\section{DISCUSSION}

In this study, the effect of Etomidate and Propofol drugs on dementia and delirium in patients over 60 years of age underwent orthopaedic surgery are evaluated. The results of the current study showed that the dementia of the first and third day after surgery was lower in Propofol group, which may be due to the short-term effect of Propofol and lack of high levels storage in body tissue. Therefore, it seems that the use of Propofol reduce the chance of developing delirium on the first day after surgery.(15) Delirium, depression and dementia are among the most common psychiatric disorders that occur in elderly patients under orthopaedic surgery.(5) Of these disorders, delirium is more important.(6) There are various theories about delirium pathophysiology, $(5,6)$ but the important issue is that delirium and postoperative dementia can disrupt the postoperative recovery and prolong the hospitalization period.(16) The findings of this study showed that the use of Propofol decreased the chance of Delirium. Also, Grati et al. evaluate the two drugs in patients undergoing ECT achieved similar results.(17) Our findings showed that MMSE scores of patients receiving Propofol were higher. In this regard, Brun and Martin that examined the cognitive activities of patients undergoing neurosurgery 
found verbal and written obedience in Propofol and fentanyl recipients was significantly shorter than those receiving ephedrine and fentanyl.(18) As mentioned, this can be due to short half-life and low accumulation of Propofol in the body.

The findings of this study showed that haemodynamic changes in Propofol group were higher than those receiving the Etomidate, indicating a greater effect of the Propofol on the cardiovascular system. Grati et al. showed that there were no haemodynamic changes (especially blood pressure) between Propofol and Etomidate recipients.(17) Perhaps, this difference in results is due to the difference in the brand of Propofol and Etomidate used in two studies, as well as the difference in age, duration, and type of surgery in patients. Also, in orthopaedic patients, reducing intravascular volume is more due to bleeding and dehydration.(19,20) Perhaps, the difference in sample size of two studies is a reason for the difference in results. Izard et al. found that the patients under treatment with Propofol had a greater reducing diastolic blood pressure than patients under treatment with Etomidate in emergency surgeries.(21) Which was consistent with the results of this study. Aggarwal et al. showed that due to less haemodynamic instability, Etomidate was the better choice for general anaesthesia than Propofol,(22) which contrasted with the present study. This difference is probably due to different doses of Etomidate and Propofol in two studies, as well as differences in the age. In the Aggarwal et al. Study, individuals aged 18-65 years were studied, while in our study, people over 60 years of age were in the study subjects. ${ }^{(22)}$

\section{CONCLUSIONS}

Use of Propofol for orthopaedic surgery in elderly patients decreases the chance of delirium and increases the amount of bleeding.

\section{ACKNOWLEDGEMENT}

The authors thank all patients who participated in this study, the research officials of Rafsanjan University of Medical Sciences and staff of Ali-Ebn Abi Taleb Hospital.

\section{REFERENCES}

[1] Afsharkohan J, Koolivand S. Structured study of the quality of life in the elderly in Iran (2004-13). Iranian Journal of Ageing 2015;10(3):192-201.

[2] Mirzaie M, Darabi S. Population aging in Iran and rising health care costs. Salmand: Iranian Journal of Ageing 2017;12(2):156-69.

[3] Darabi S, Torabi F. Analysis and comparison of aging population in Europe and Asia during 1950 to 2015. Salmand 2017;12(1):30-43.

[4] Fong TG, Davis D, Growdon ME, et al. The interface between delirium and dementia in elderly adults. Lancet Neurol 2015;14(8):823-32.
[5] Kyziridis TC. Post-operative delirium after hip fracture treatment - a review of the current literature. Psychosoc Med 2006;3:Doc01.

[6] Clayer M, Bruckner J. Occult hypoxia after femoral neck fracture and elective hip surgery. Clin Orthop Relat Res 2000;(370):265-71.

[7] Beiranvand A, Khoshkneb MF, Ashayeri H, et al. Comparison between prevalence of delirium after hip surgery and general surgery in hospitalized female elderly. Journal of Geriatric Nursing 2014;1(1):83-93.

[8] Townsend CM, Beauchamp RD. Sabiston's Textbook of Surgery. $19^{\text {th }}$ edn. Saunders 2012.

[9] Liu Y, Ma L, Gao M, et al. Dexmedetomidine reduces postoperative delirium after joint replacement in elderly patients with mild cognitive impairment. Aging Clin Exp Res 2016;28(4):729-36.

[10] Bauer J, Hageman I, Dam H, et al. Comparison of propofol and thiopental as anesthetic agents for electroconvulsive therapy: a randomized, blinded comparison of seizure duration, stimulus charge, clinical effect and cognitive side effects. J ECT 2009;25(2):85-90.

[11] Van Rompaey B, Schuurmans MJ, Shortridge-Baggett LM, et al. A comparison of the CAM-ICU and the NEECHAM Confusion Scale in intensive care delirium assessment: an observational study in non-intubated patients. Critical Care (London, England) 2008;12(1):R16.

[12] Van Gemert La, Schuurmans MJ. The Neecham Confusion Scale and the Delirium Observation Screening Scale: capacity to discriminate and ease of use in clinical practice. BMC Nurs 2007;6(1):3.

[13] Pangman VC, Sloan J, Guse L. An examination of psychometric properties of the mini-mental state examination and the standardized mini-mental state examination: implications for clinical practice. Applied Nursing Research: ANR 2000;13(4):209-13.

[14] Klimek L, Bergmann KC, Biedermann T, et al. Visual analogue scales (VAS): Measuring instruments for the documentation of symptoms and therapy monitoring in cases of allergic rhinitis in everyday health care: Position Paper of the German Society of Allergology (AeDA) and the German Society of Allergy and Clinical Immunology (DGAKI), ENT Section, in collaboration with the working group on Clinical Immunology, Allergology and Environmental Medicine of the German Society of Otorhinolaryngology, Head and Neck Surgery (DGHNOKHC). Allergo J Int 2017;26(1):16-24.

[15] Brunner LS, Siddarth PS. Text book of Medical- surgical nursing. Philadelphia: JB Lippincott 2012.

[16] Sohrabi M, Jannati Y, Bagheri NM, et al. Incidence of delirium and associated factors before open heart surgery. JGBFNM 2013;10(1):33-42.

[17] Grati L, Louzi M, Nasr K, et al. Compared effects of etomidate and propofol for anaesthesia during electroconvulsive therapy. Presse Med 2005;34(4):2824.

[18] Brun TH, Martin C. Comparative study of recovery in neurosurgery after continuous perfusion of etomidate or propofol. Cah Anesthesiol 1995;43(1):27-30.

[19] Oberweis BS, Nukala S, Rosenberg A, et al. Thrombotic and bleeding complications after orthopaedic surgery. Am Heart J 2013;165(3):427-33.e1. 
[20] Sambandam B, Batra S, Gupta R, et al. Blood conservation strategies in orthopaedic surgeries: a review. J Clin Orthop Trauma 2013;4(4):164-70.

[21] Izard P, Coustets B, Olivier M, et al. Propofol and etomidate: a comparative study in emergency anaesthesia in ASA I and II patients. Cah Anesthesiol 1991;39(6):399-403.
[22] Aggarwal S, Goyal VK, Chaturvedi SK, et al. A comparative study between propofol and etomidate in patients under general anaesthesia. Braz J Anesthesiol 2016;66(3):237-41. 